\title{
Colinear Coloring on Graphs ${ }^{\star}$
}

\author{
Kyriaki Ioannidou and Stavros D. Nikolopoulos \\ Department of Computer Science, University of Ioannina \\ GR-45110 Ioannina, Greece \\ \{kioannid, stavros\}@cs.uoi.gr
}

\begin{abstract}
Motivated by the definition of linear coloring on simplicial complexes, recently introduced in the context of algebraic topology, and the framework through which it was studied, we introduce the colinear coloring on graphs. We provide an upper bound for the chromatic number $\chi(G)$, for any graph $G$, and show that $G$ can be colinearly colored in polynomial time by proposing a simple algorithm. The colinear coloring of a graph $G$ is a vertex coloring such that two vertices can be assigned the same color, if their corresponding clique sets are associated by the set inclusion relation (a clique set of a vertex $u$ is the set of all maximal cliques containing $u$ ); the colinear chromatic number $\lambda(G)$ of $G$ is the least integer $k$ for which $G$ admits a colinear coloring with $k$ colors. Based on the colinear coloring, we define the $\chi$-colinear and $\alpha$ colinear properties and characterize known graph classes in terms of these properties.
\end{abstract}

Keywords: Colinear coloring, chromatic number, chordal graphs, threshold graphs, quasi-threshold graphs, algorithms, complexity.

\section{Introduction}

A colinear coloring of a graph $G$ is a coloring of its vertices such that two vertices are assigned different colors, if their corresponding clique sets are not associated by the set inclusion relation; a clique set of a vertex $u$ is the set of all maximal cliques in $G$ containing $u$. The colinear chromatic number $\lambda(G)$ of $G$ is the least integer $k$ for which $G$ admits a colinear coloring with $k$ colors.

Motivated by the definition of linear coloring on simplicial complexes associated to graphs, first introduced by Civan and Yalçin [5] in the context of algebraic topology, we studied linear colorings on simplicial complexes which can be represented by a graph. In particular, we studied the linear coloring problem on a simplicial complex, namely independence complex $\mathcal{I}(G)$ of a graph $G$. The independence complex $\mathcal{I}(G)$ of a graph $G$ can always be represented by a graph and, more specifically, is identical to the complement graph $\bar{G}$ of the graph $G$; indeed, the facets of $\mathcal{I}(G)$ are exactly the maximal cliques of $\bar{G}$. The outcome of this study was the definition of the colinear coloring of a graph $G$; the colinear

\footnotetext{
* This research is co-financed by E.U.-European Social Fund (75\%) and the Greek Ministry of Development-GSRT (25\%).
} 
coloring of a graph $G$ is a coloring of $G$ such that for any set of vertices taking the same color, the collection of their clique sets can be linearly ordered by inclusion. Note that, the two definitions cannot always be considered as identical since not in all cases a simplicial complex can be represented by a graph; such an example is the neighborhood complex $\mathcal{N}(G)$ of a graph $G$. Recently, Civan and Yalçin [5] studied the linear coloring of the neighborhood complex $\mathcal{N}(G)$ of a graph $G$ and proved that the linear chromatic number of $\mathcal{N}(G)$ gives an upper bound for the chromatic number $\chi(G)$ of the graph $G$. This approach lies in a general framework met in algebraic topology.

In the context of algebraic topology, one can find much work done on providing boundaries for the chromatic number of an arbitrary graph $G$, by examining the topology of the graph through different simplicial complexes associated to the graph. This domain was motivated by Kneser's conjecture, which was posed in 1955 , claiming that "if we split the $n$-subsets of a $(2 n+k)$-element set into $k+1$ classes, one of the classes will contain two disjoint $n$-subsets" 9 . Kneser's conjecture was first proved by Lovász in 1978, with a proof based on graph theory, by rephrasing the conjecture into "the chromatic number of Kneser's graph $K G_{n, k}$ is $k+2$ " [10. Many more topological and combinatorial proofs followed the interest of which extends beyond the original conjecture [13. Although Kneser's conjecture is concerned with the chromatic numbers of certain graphs (Kneser graphs), the proof methods that are known provide lower bounds for the chromatic number of any graph 11. Thus, this initiated the application of topological tools in studying graph theory problems and more particularly in graph coloring problems [6].

The interest to provide boundaries for the chromatic number $\chi(G)$ of an arbitrary graph $G$ through the study of different simplicial complexes associated to $G$, which is found in algebraic topology bibliography, drove the motivation for defining the colinear coloring on the graph $G$ and studying the relation between the chromatic number $\chi(G)$ and the colinear chromatic number $\lambda(\bar{G})$. We show that for any graph $G, \lambda(\bar{G})$ is an upper bound for $\chi(G)$. The interest of this result lies on the fact that we present a colinear coloring algorithm that can be applied to any graph $G$ and provides an upper bound $\lambda(\bar{G})$ for the chromatic number of the graph $G$, i.e. $\chi(G) \leq \lambda(\bar{G})$; in particular, it provides a proper vertex coloring of $G$ using $\lambda(\bar{G})$ colors. Additionally, recall that a known lower bound for the chromatic number of any graph $G$ is the clique number $\omega(G)$ of $G$, i.e. $\chi(G) \geq \omega(G)$. Motivated by the definition of perfect graphs, for which $\chi\left(G_{A}\right)=\omega\left(G_{A}\right)$ holds $\forall A \subseteq V(G)$, it was interesting to study those graphs for which the equality $\chi(G)=\lambda(\bar{G})$ holds, and even more those graphs for which this equality holds for every induced subgraph.

In this paper, we first introduce the colinear coloring of a graph $G$ and study the relation between the colinear coloring of $\bar{G}$ and the proper vertex coloring of $G$. We prove that, for any graph $G$, a colinear coloring of $\bar{G}$ is a proper vertex coloring of $G$ and, thus, $\lambda(\bar{G})$ is an upper bound for $\chi(G)$, i.e. $\chi(G) \leq \lambda(\bar{G})$. We present a colinear coloring algorithm that can be applied to any graph $G$. Motivated by these results and the Perfect Graph Theorem [7, we study those 
graphs for which the equality $\chi(G)=\lambda(\bar{G})$ holds for every induce subgraph and characterize known graph classes in terms of the $\chi$-colinear and the $\alpha$-colinear properties. A graph $G$ has the $\chi$-colinear property if its chromatic number $\chi(G)$ equals to the colinear chromatic number $\lambda(\bar{G})$ of its complement graph $\bar{G}$, and the equality holds for every induced subgraph of $G$, i.e. $\chi\left(G_{A}\right)=\lambda\left(\bar{G}_{A}\right), \forall A \subseteq V(G)$; a graph $G$ has the $\alpha$-colinear property if its stability number $\alpha(G)$ equals to its colinear chromatic number $\lambda(G)$, and the equality holds for every induced subgraph of $G$, i.e. $\alpha\left(G_{A}\right)=\lambda\left(G_{A}\right), \forall A \subseteq V(G)$. Note that the stability number $\alpha(G)$ of a graph $G$ is the greatest integer $r$ for which $G$ contains an independent set of size $r$. We show that the class of threshold graphs is characterized by the $\chi$-colinear property and the class of quasi-threshold graphs is characterized by the $\alpha$-colinear property.

\section{Preliminaries}

Let $G$ be a finite undirected graph with no loops or multiple edges. We denote by $V(G)$ and $E(G)$ the vertex set and edge set of $G$. The subgraph of a graph $G$ induced by a subset $S$ of vertices of $G$ is denoted by $G[S]$.

An edge is a pair of distinct vertices $x, y \in V(G)$, and is denoted by $x y$ if $G$ is an undirected graph and by $\overrightarrow{x y}$ if $G$ is a directed graph. For a set $A \subseteq V(G)$ of vertices of the graph $G$, the subgraph of $G$ induced by $A$ is denoted by $G_{A}$. Additionally, the cardinality of a set $A$ is denoted by $|A|$. The set $N(v)=\{u \in$ $V(G): u v \in E(G)\}$ is called the open neighborhood of the vertex $v \in V(G)$ in $G$, sometimes denoted by $N_{G}(v)$ for clarity reasons. The set $N[v]=N(v) \cup\{v\}$ is called the closed neighborhood of the vertex $v \in V(G)$ in $G$.

The greatest integer $r$ for which a graph $G$ contains an independent set of size $r$ is called the independence number or otherwise the stability number of $G$ and is denoted by $\alpha(G)$. The cardinality of the vertex set of the maximum clique in $G$ is called the clique number of $G$ and is denoted by $\omega(G)$. A proper vertex coloring of a graph $G$ is a coloring of its vertices such that no two adjacent vertices are assigned the same color. The chromatic number $\chi(G)$ of $G$ is the least integer $k$ for which $G$ admits a proper vertex coloring with $k$ colors. For the numbers $\omega(G)$ and $\chi(G)$ of an arbitrary graph $G$ the inequality $\omega(G) \leq \chi(G)$ holds. In particularly, $G$ is a perfect graph if the equality $\omega\left(G_{A}\right)=\chi\left(G_{A}\right)$ holds $\forall A \subseteq V(G)$.

Next, definitions of some graph classes mentioned throughout the paper follow. A graph is called a chordal graph if it does not contain an induced subgraph isomorphic to a chordless cycle of four or more vertices. A graph is called a $\mathrm{co}$ chordal graph if it is the complement of a chordal graph [7]. A hole is a chordless cycle $C_{n}$ if $n \geq 5$; the complement of a hole is an antihole. A graph $G$ is a split graph if there is a partition of the vertex set $V(G)=K+I$, where $K$ induces a clique in $G$ and $I$ induces an independent set. Split graphs are characterized as $\left(2 K_{2}, C_{4}, C_{5}\right)$-free. Threshold graphs are defined as those graphs where stable subsets of their vertex sets can be distinguished by using a single linear inequality. Threshold graphs were introduced by Chvátal and Hammer [4] and characterized 
as $\left(2 K_{2}, P_{4}, C_{4}\right)$-free. Quasi-threshold graphs are characterized as the $\left(P_{4}, C_{4}\right)$ free graphs and are also known in the literature as trivially perfect graphs [7], [12. For more details on basic definitions in graph theory refer to [2], 7].

\section{Colinear Coloring on Graphs}

In this section we define the colinear coloring of a graph $G$, and we prove some properties of the colinear coloring of $G$. It is worth noting that these properties have been also proved for the linear coloring of the neighborhood complex $\mathcal{N}(G)$ in [5].

Definition 1. Let $G$ be a graph and let $v \in V(G)$. The clique set of a vertex $v$ is the set of all maximal cliques of $G$ containing $v$ and is denoted by $\mathcal{C}_{G}(v)$.

Definition 2. Let $G$ be a graph and let $k$ be an integer. A surjective map $\kappa$ : $V(G) \rightarrow\{1,2, \ldots, k\}$ is called a $k$-colinear coloring of $G$ if the collection $\left\{\mathcal{C}_{G}(v)\right.$ : $\kappa(v)=i\}$ is linearly ordered by inclusion for all $i \in\{1,2, \ldots, k\}$, where $\mathcal{C}_{G}(v)$ is the clique set of $v$, or, equivalently, for two vertices $v, u \in V(G)$, if $\kappa(v)=\kappa(u)$ then either $\mathcal{C}_{G}(v) \subseteq \mathcal{C}_{G}(u)$ or $\mathcal{C}_{G}(v) \supseteq \mathcal{C}_{G}(u)$. The least integer $k$ for which $G$ is $k$-colinear colorable is called the colinear chromatic number of $G$ and is denoted by $\lambda(G)$.

Next, we study the colinear coloring on graphs and its association to the proper vertex coloring. In particular, we show that for any graph $G$ the colinear chromatic number of $\bar{G}$ is an upper bound for $\chi(G)$.

Proposition 1. Let $G$ be a graph. If $\kappa: V(G) \rightarrow\{1,2, \ldots, k\}$ is a $k$-colinear coloring of $\bar{G}$, then $\kappa$ is a coloring of the graph $G$.

Proof. Let $G$ be a graph and let $\kappa: V(G) \rightarrow\{1,2, \ldots, k\}$ be a $k$-colinear coloring of $\bar{G}$. From Definition [2, we have that for any two vertices $v, u \in V(G)$, if $\kappa(v)=\kappa(u)$ then either $\mathcal{C}_{\bar{G}}(v) \subseteq \mathcal{C}_{\bar{G}}(u)$ or $\mathcal{C}_{\bar{G}}(v) \supseteq \mathcal{C}_{\bar{G}}(u)$ holds. Without loss of generality, assume that $\mathcal{C}_{\bar{G}}(v) \subseteq \mathcal{C}_{\bar{G}}(u)$ holds. Consider a maximal clique $C \in \mathcal{C}_{\bar{G}}(v)$. Since $\mathcal{C}_{\bar{G}}(v) \subseteq \mathcal{C}_{\bar{G}}(u)$, we have $C \in \mathcal{C}_{\bar{G}}(u)$. Thus, both $u, v \in C$ and therefore $u v \in E(\bar{G})$ and $u v \notin E(G)$. Hence, any two vertices assigned the same color in a $k$-colinear coloring of $\bar{G}$ are not neighbors in $G$. Concluding, any $k$-colinear coloring of $\bar{G}$ is a coloring of $G$.

It is therefore straightforward to conclude the following.

Corollary 1. For any graph $G, \lambda(\bar{G}) \geq \chi(G)$.

In Figure 1 we depict a colinear coloring of the well known graphs $2 K_{2}, C_{4}$ and $P_{4}$, using the least possible colors, and show the relation between the chromatic number $\chi(G)$ of each graph $G \in\left\{2 K_{2}, C_{4}, P_{4}\right\}$ and the colinear chromatic number $\lambda(\bar{G})$. 

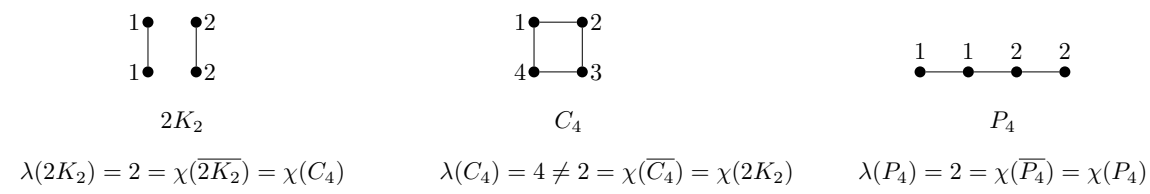

Fig. 1. Illustrating a colinear coloring of the graphs $2 K_{2}, C_{4}$ and $P_{4}$ with the least possible colors

Proposition 2. Let $G$ be a graph. A coloring $\kappa: V(G) \rightarrow\{1,2, \ldots, k\}$ of $G$ is a $k$-colinear coloring of $G$ if and only if either $N_{G}[u] \subseteq N_{G}[v]$ or $N_{G}[u] \supseteq N_{G}[v]$ holds in $G$, for every $u, v \in V(G)$ with $\kappa(u)=\kappa(v)$.

Proof. Let $G$ be a graph and let $\kappa: V(G) \rightarrow\{1,2, \ldots, k\}$ be a $k$-colinear coloring of $G$. We will show that either $N_{G}[u] \subseteq N_{G}[v]$ or $N_{G}[u] \supseteq N_{G}[v]$ holds in $G$ for every $u, v \in V(G)$ with $\kappa(u)=\kappa(v)$. Consider two vertices $v, u \in V(G)$, such that $\kappa(u)=\kappa(v)$. Since $\kappa$ is a colinear coloring of $G$, we have either $\mathcal{C}_{G}(u) \subseteq \mathcal{C}_{G}(v)$ or $\mathcal{C}_{G}(u) \supseteq \mathcal{C}_{G}(v)$ holds. Without loss of generality, assume that $\mathcal{C}_{G}(u) \subseteq \mathcal{C}_{G}(v)$. We will show that $N_{G}[u] \subseteq N_{G}[v]$ holds in $G$. Assume the opposite. Thus, a vertex $z \in V(G)$ exists, such that $z \in N_{G}[u]$ and $z \notin N_{G}[v]$ and, thus, $z u \in E(G)$ and $z v \notin E(G)$. Now consider a maximal clique $C$ in $G$ which contains $z$ and $u$. Since $z v \notin E(G)$, it follows that $v \notin C$. Thus, there exists a maximal clique $C$ in $G$ such that $C \in \mathcal{C}_{G}(u)$ and $C \notin \mathcal{C}_{G}(v)$, which is a contradiction to our assumption that $\mathcal{C}_{G}(u) \subseteq \mathcal{C}_{G}(v)$. Therefore, $N_{G}[u] \subseteq N_{G}[v]$ holds in $G$.

Let $G$ be a graph and let $\kappa: V(G) \rightarrow\{1,2, \ldots, k\}$ be a coloring of $G$. Assume now that either $N_{G}[u] \subseteq N_{G}[v]$ or $N_{G}[u] \supseteq N_{G}[v]$ holds in $G$, for every $u, v \in$ $V(G)$ with $\kappa(u)=\kappa(v)$. We will show that the coloring $\kappa$ of $G$ is a $k$-colinear coloring of $G$. Without loss of generality, assume that $N_{G}[u] \subseteq N_{G}[v]$ holds in $G$, and we will show that $\mathcal{C}_{G}(u) \subseteq \mathcal{C}_{G}(v)$. Assume the opposite. Thus, a maximal clique $C$ exists in $G$, such that $C \in \mathcal{C}_{G}(u)$ and $C \notin \mathcal{C}_{G}(v)$. Consider now a vertex $z \in V(G)(z \neq v)$, such that $z \in C$ and $z v \notin E(G)$. Such a vertex exists since $C$ is maximal in $G$ and $C \notin \mathcal{C}_{G}(v)$. Thus, $z v \notin E(G)$ and either $z u \in E(G)$ or $z=u$, which is a contradiction to our assumption that $N_{G}[u] \subseteq N_{G}[v]$.

\section{An Algorithm for Colinear Coloring}

In this section we present a polynomial time algorithm for colinear coloring which can be applied to any graph $G$, and provides an upper bound for $\chi(G)$. Although we have introduced colinear coloring through Definition 2, in our algorithm we exploit the property proved in Proposition 2 , since the problem of finding all maximal cliques of a graph $G$ is not polynomially solvable on general graphs. Before describing our algorithm, we first construct a directed acyclic graph (DAG) $D_{G}$ of a graph $G$, which we call $D A G$ associated to the graph $G$, and we use it in the proposed algorithm.

The DAG $D_{G}$ associated to the graph $G$. Let $G$ be a graph. We first compute the closed neighborhood $N_{G}[v]$ of each vertex $v$ of $G$ and, then, we 
construct the following directed acyclic graph $D$, which depicts all inclusion relations among the vertices' closed neighborhoods: $V(D)=V(G)$ and $E(D)=$ $\left\{\overrightarrow{x y}: x, y \in V(D)\right.$ and $\left.N_{G}[x] \subseteq N_{G}[y]\right\}$, where $\overrightarrow{x y}$ is a directed edge from $x$ to $y$. In the case where the equality $N_{G}[x]=N_{G}[y]$ holds, we choose to add one of the two edges so that the resulting graph $D$ is acyclic. To achieve this, we consider a partition of the vertex set $V(G)$ into the sets $S_{1}, S_{2}, \ldots, S_{\ell}$, such that for any $i \in\{1,2, \ldots, \ell\}$ vertices $x$ and $y$ belong to a set $S_{i}$ if and only if $N_{G}[x]=N_{G}[y]$. For vertices $x$ and $y$ belonging to the same set $S_{i}$ we add the edge $\overrightarrow{x y}$ if and only if $x<y$. For vertices $x$ and $y$ belonging to different sets $S_{i}$ and $S_{j}$ respectively, we add the edge $\overrightarrow{x y}$ if and only if $N_{G}[x] \subset N_{G}[y]$. It is easy to see that the resulting graph $D$ is unique up to isomorphism.

Additionally, it is easy to see that $D$ is a transitive directed acyclic graph. Indeed, by definition $D$ is constructed on a partially ordered set of elements $(V(D), \leq)$, such that for some $x, y \in V(D), x \leq y \Leftrightarrow N_{G}[x] \subseteq N_{G}[y]$. Throughout the paper we refer to the constructed directed acyclic graph as the DAG associated to the graph $G$ and denote it by $D_{G}$.

The algorithm for colinear coloring. The proposed algorithm computes a colinear coloring and the colinear chromatic number of a graph $G$. The algorithm works as follows:

(i) compute the closed neighborhood set of every vertex of $G$ and, then, find the inclusion relations among the neighborhood sets and construct the DAG $D_{G}$ associated to the graph $G$.

(ii) find a minimum path cover $\mathcal{P}\left(D_{G}\right)$, and its size $\rho\left(D_{G}\right)$, of the transitive DAG $D_{G}$ (e.g. see [1, [8]).

(iii) assign a color $\kappa(v)$ to each vertex $v \in V\left(D_{G}\right)$, such that vertices belonging to the same path of $\mathcal{P}\left(D_{G}\right)$ are assigned the same color and vertices of different paths are assigned different colors; this is a surjective map $\kappa$ : $V\left(D_{G}\right) \rightarrow\left[\rho\left(D_{G}\right)\right]$.

(iv) return the value $\kappa(v)$ for each vertex $v \in V\left(D_{G}\right)$ and the size $\rho\left(D_{G}\right)$ of the minimum path cover of $D_{G} ; \kappa$ is a colinear coloring of $G$ and $\rho\left(D_{G}\right)$ equals the colinear chromatic number $\lambda(G)$ of $G$.

Correctness of the algorithm. Let $G$ be a graph and let $D_{G}$ be the DAG associated to the graph $G$, which is unique up to isomorphism. Consider the value $\kappa(v)$ for each vertex $v \in V\left(D_{G}\right)$ returned by the algorithm and the size $\rho\left(D_{G}\right)$ of a minimum path cover of $D_{G}$. We show that the surjective map $\kappa$ : $V\left(D_{G}\right) \rightarrow\left[\rho\left(D_{G}\right)\right]$ is a colinear coloring of the vertices of $G$, and prove that the size $\rho\left(D_{G}\right)$ of a minimum path cover $\mathcal{P}\left(D_{G}\right)$ of the DAG $D_{G}$ is equal to the colinear chromatic number $\lambda(G)$ of the graph $G$.

Proposition 3. Let $G$ be a graph and let $D_{G}$ be the DAG associated to the graph $G$. A colinear coloring of the graph $G$ can be obtained by assigning a particular color to all vertices of each path of a path cover of the $D A G D_{G}$. Moreover, the size $\rho\left(D_{G}\right)$ of a minimum path cover $\mathcal{P}\left(D_{G}\right)$ of the $D A G D_{G}$ equals to the colinear chromatic number $\lambda(G)$ of the graph $G$. 
Proof. Let $G$ be a graph, $D_{G}$ be the DAG associated to $G$, and let $\mathcal{P}\left(D_{G}\right)$ be a minimum path cover of $D_{G}$. The size $\rho\left(D_{G}\right)$ of the DAG $D_{G}$, equals to the minimum number of directed paths in $D_{G}$ needed to cover the vertices of $D_{G}$ and, thus, the vertices of $G$. Now, consider a coloring $\kappa: V\left(D_{G}\right) \rightarrow\{1,2, \ldots, k\}$ of the vertices of $D_{G}$, such that vertices belonging to the same path are assigned the same color and vertices of different paths are assigned different colors. Therefore, we have $\rho\left(D_{G}\right)$ colors and $\rho\left(D_{G}\right)$ sets of vertices, one for each color. For every set of vertices belonging to the same path, their corresponding closed neighborhood sets can be linearly ordered by inclusion. Indeed, consider a path in $D_{G}$ with vertices $\left\{v_{1}, v_{2}, \ldots, v_{m}\right\}$ and edges $\overrightarrow{v_{i} v_{i+1}}$ for $i \in\{1,2, \ldots, m\}$. From the construction of $D_{G}$, it holds that $\forall i, j \in\{1,2, \ldots, m\}, \overrightarrow{v_{i} v_{j}} \in E\left(D_{G}\right) \Leftrightarrow$ $N_{G}\left[v_{i}\right] \subseteq N_{G}\left[v_{j}\right]$. In other words, the corresponding neighborhood sets of the vertices belonging to a path in $D_{G}$ are linearly ordered by inclusion. Thus, the coloring $\kappa$ of the vertices of $D_{G}$ gives a colinear coloring of $G$. This colinear coloring $\kappa$ is optimal, uses $k=\rho\left(D_{G}\right)$ colors, and gives the colinear chromatic number $\lambda(G)$ of the graph $G$. Indeed, suppose that there exists a different colinear coloring $\kappa^{\prime}: V\left(D_{G}\right) \rightarrow\left[k^{\prime}\right]$ of $G$ using $k^{\prime}$ colors, such that $k^{\prime}<k$. For every color given in $\kappa^{\prime}$, consider a set consisted of the vertices assigned that color. It is true that for the vertices belonging to the same set, their neighborhood sets are linearly ordered by inclusion. Therefore, these vertices can belong to the same path in $D_{G}$. Thus, each set of vertices in $G$ corresponds to a path in $D_{G}$ and, additionally, all vertices of $G$ (and therefore of $D_{G}$ ) are covered. This is a path cover of $D_{G}$ of size $\rho^{\prime}\left(D_{G}\right)=k^{\prime}<k=\rho\left(D_{G}\right)$, which is a contradiction since $\mathcal{P}\left(D_{G}\right)$ is a minimum path cover of $D_{G}$. Therefore, we conclude that the colinear coloring $\kappa: V\left(D_{G}\right) \rightarrow\left[\rho\left(D_{G}\right)\right]$ is optimal and, hence, $\rho\left(D_{G}\right)=\lambda(G)$.

Complexity of the algorithm. Let $G$ be a graph, $V(G)=n, E(G)=m$, and let $D_{G}$ be the DAG associated to the graph $G$. Step (i) of the algorithm, which includes the construction of the DAG $D_{G}$, takes $O(n m)$ time. In particular, it takes $O(\mathrm{~nm})$ time to compute the closed neighborhood set of every vertex of $G$, $O(n m)$ time to find the inclusion relations among the neighborhood sets, and $O(n+m)$ time to construct the DAG $D_{G}$. Note that, we only need to check pairs of vertices that are connected by an edge in $G$. Step (ii) computes a minimum path cover in the transitive DAG $D_{G}$; the problem is known to be polynomially solvable, since it can be reduced to the maximum matching problem in a bipartite graph formed from the transitive DAG [1. The maximum matching problem in a bipartite graph takes $O((m+n) \sqrt{n})$ time, due to an algorithm by Hopcroft and Karp [8]. Finally, both Steps (iii) and (iv) can be executed in $O(n)$ time. Therefore, the complexity of the algorithm is $O(n m+n \sqrt{n})$.

\section{Graphs Having the $\chi$-Colinear and $\alpha$-Colinear Properties}

In Section 3 we showed that for any graph $G$, the colinear chromatic number $\lambda(\bar{G})$ of $\bar{G}$ is an upper bound for the chromatic number $\chi(G)$ of $G$, i.e. $\chi(G) \leq$ 
$\lambda(\bar{G})$. Recall that a known lower bound for the chromatic number of $G$ is the clique number $\omega(G)$ of $G$, i.e. $\chi(G) \geq \omega(G)$. Motivated by the Perfect Graph Theorem [7, in this section we exploit our results on colinear coloring and we study those graphs for which the equality $\chi(G)=\lambda(\bar{G})$ holds for every induced subgraph. The outcome of this study was the definition of the following two graph properties and the characterization of known graph classes in terms of these properties.

○ $\chi$-colinear property. A graph $G$ has the $\chi$-colinear property if for every induced subgraph $G_{A}$ of the graph $G, \chi\left(G_{A}\right)=\lambda\left(\bar{G}_{A}\right), A \subseteq V(G)$.

○ $\alpha$-colinear property. A graph $G$ has the $\alpha$-colinear property if for every induced subgraph $G_{A}$ of a graph $G, \alpha\left(G_{A}\right)=\lambda\left(G_{A}\right), A \subseteq V(G)$.

Next, we show that the class of threshold graphs is characterized by the $\chi$ colinear property and the class of quasi-threshold graphs is characterized by the $\alpha$-colinear property. We also show that any graph that has the $\chi$-colinear property is perfect; actually, we show that any graph that has the $\chi$-colinear property is a co-chordal graph, and any graph that has the $\alpha$-colinear property is a chordal graph. We first give some definitions and show some interesting results.

Definition 3. The edge uv of a graph $G$ is called actual if neither $N_{G}[u] \subseteq N_{G}[v]$ nor $N_{G}[u] \supseteq N_{G}[v]$. The set of all actual edges of $G$ will be denoted by $E_{\alpha}(G)$.

Definition 4. A graph $G$ is called quasi-threshold if it has no induced subgraph isomorphic to $a C_{4}$ or a $P_{4}$ or, equivalently, if it contains no actual edges.

More details on actual edges and characterizations of quasi-threshold graphs through a classification of their edges can be found in [12. The following result directly follows from Definition 3 and Proposition 2 .

Proposition 4. Let $\kappa: V(G) \rightarrow\{1,2, \ldots, k\}$ be a $k$-colinear coloring of the graph $G$. If the edge $u v \in E(G)$ is an actual edge of $G$, then $\kappa(u) \neq \kappa(v)$.

Based on Definition 3 the $\chi$-colinear property and Proposition 5.1 , we prove the following result.

Proposition 5. Let $G$ be a graph and let $F$ be the graph such that $V(F)=$ $V(G)$ and $E(F)=E(G) \cup E_{\alpha}(\bar{G})$. The graph $G$ has the $\chi$-colinear property if $\chi\left(G_{A}\right)=\omega\left(F_{A}\right), \forall A \subseteq V(G)$.

Proof. Let $G$ be a graph and let $F$ be a graph such that $V(F)=V(G)$ and $E(F)=E(G) \cup E_{\alpha}(\bar{G})$, where $E_{\alpha}(\bar{G})$ is the set of all actual edges of $\bar{G}$. By definition, $G$ has the $\chi$-colinear property if $\chi\left(G_{A}\right)=\lambda\left(\bar{G}_{A}\right), \forall A \subseteq V(G)$. It suffices to show that $\lambda\left(\bar{G}_{A}\right)=\omega\left(F_{A}\right), \forall A \subseteq V(G)$. From Definition 2] it is easy to see that two vertices which are not connected by an edge in $\bar{G}_{A}$ belong necessarily to different cliques and, thus, they cannot receive the same color in a colinear coloring of $\bar{G}_{A}$. In other words, the vertices which are connected by an 
edge in $G_{A}$ cannot take the same color in a colinear coloring of $\bar{G}_{A}$. Moreover, from Proposition 4 vertices which are endpoints of actual edges in $\bar{G}_{A}$ cannot take the same color in a colinear coloring of $\bar{G}_{A}$.

Next, we construct the graph $F_{A}$ with vertex set $V\left(F_{A}\right)=V\left(G_{A}\right)$ and edge set $E\left(F_{A}\right)=E\left(G_{A}\right) \cup E_{\alpha}\left(\bar{G}_{A}\right)$, where $E_{\alpha}\left(\bar{G}_{A}\right)$ is the set of all actual edges of $\bar{G}_{A}$. Every two vertices in $F_{A}$, which have to take a different color in a colinear coloring of $\bar{G}_{A}$ are connected by an edge. Thus, the size of the maximum clique in $F_{A}$ equals to the size of the maximum set of vertices which pairwise must take a different color in $\bar{G}_{A}$, i.e. $\omega\left(F_{A}\right)=\lambda\left(\bar{G}_{A}\right)$ holds for all $A \subseteq V(G)$. Concluding, $G$ has the $\chi$-colinear property if $\chi\left(G_{A}\right)=\omega\left(F_{A}\right), \forall A \subseteq V(G)$.

Taking into consideration Proposition 5 and the structure of the edge set $E(F)=$ $E(G) \cup E_{\alpha}(\bar{G})$ of the graph $F$, it is easy to see that $E(F)=E(G)$ if $\bar{G}$ has no actual edges. Actually, this will be true for all induced subgraphs, since if $G$ is a quasi-threshold graph then $G_{A}$ is also a quasi-threshold graph for all $A \subseteq V(G)$. Thus, $\chi\left(G_{A}\right)=\omega\left(F_{A}\right), \forall A \subseteq V(G)$. Therefore, the following result holds.

Corollary 2. Let $G$ be a graph. If $\bar{G}$ is quasi-threshold, then $G$ has the $\chi$ colinear property.

From Corollary 2 we obtain a more interesting result.

\section{Proposition 6. Any threshold graph has the $\chi$-colinear property.}

Proof. Let $G$ be a threshold graph. It has been proved that an undirected graph $G$ is a threshold graph if and only if $G$ and its complement $\bar{G}$ are quasi-threshold graphs 12. From Corollary 2, if $\bar{G}$ is quasi-threshold then $G$ has the $\chi$-colinear property. Concluding, if $G$ is threshold, then $\bar{G}$ is quasi-threshold and thus $G$ has the $\chi$-colinear property.

However, not any graph that has the $\chi$-colinear property is a threshold graph. Indeed, Chvátal and Hammer [4] showed that threshold graphs are $\left(2 K_{2}, P_{4}, C_{4}\right)$ free and, thus, the graphs $P_{4}$ and $C_{4}$ have the $\chi$-colinear property but they are not threshold graphs (see Figure 1). We note that the proof that any threshold graph $G$ has the $\chi$-colinear property can be also obtained by showing that any coloring of a threshold graph $G$ is a colinear coloring of $\bar{G}$ by using Proposition2, the basic set theory property that $N_{G}(u)=V(G) \backslash N_{\bar{G}}[u]$, Corollary 1 and the property that $N(u) \subseteq N[v]$ or $N(v) \subseteq N[u]$ for any two vertices $u, v$ of $G$. However, Proposition 5 and Corollary 2 actually give us a stronger result since the class of quasi-threshold graphs is a superclass of the class of threshold graphs.

The following result is even more interesting, since it shows that any graph that has the $\chi$-colinear property is a perfect graph.

Proposition 7. Any graph that has the $\chi$-colinear property is a co-chordal graph.

Proof. Let $G$ be a graph that has the $\chi$-colinear property. It has been showed that a co-chordal graph is $\left(2 K_{2}\right.$, antihole)-free [7]. To show that any graph $G$ that has 
the $\chi$-colinear property is a co-chordal graph we will show that if $G$ has a $2 K_{2}$ or an antihole as induced subgraph, then $G$ is does not have the $\chi$-colinear property. Since by definition a graph $G$ has the $\chi$-colinear property if the equality $\chi\left(G_{A}\right)=\lambda\left(\bar{G}_{A}\right)$ holds for every induced subgraph $G_{A}$ of $G$, it suffices to show that the graphs $2 K_{2}$ and antihole do not have the $\chi$-colinear property.

The graph $2 K_{2}$ does not have the $\chi$-colinear property, since $\chi\left(2 K_{2}\right)=2 \neq 4=$ $\lambda\left(C_{4}\right)$; see Figure 1. Now, consider the graph $G=\bar{C}_{n}$ which is an antihole of size $n \geq 5$. We will show that $\chi(G) \neq \lambda(\bar{G})$. It follows that $\lambda(\bar{G})=\lambda\left(C_{n}\right)=n \geq 5$, i.e. if the graph $\bar{G}=C_{n}$ is to be colored colinearly, every vertex has to take a different color. Indeed, assume that a colinear coloring $\kappa: V(G) \rightarrow\{1,2, \ldots, k\}$ of $\bar{G}=C_{n}$ exists such that for some $u_{i}, u_{j} \in V(G), i \neq j, 1 \leq i, j \leq n$, $\kappa\left(u_{i}\right)=\kappa\left(u_{j}\right)$. Since $u_{i}, u_{j}$ are vertices of a hole, their neighborhoods in $\bar{G}$ are $N\left[u_{i}\right]=\left\{u_{i-1}, u_{i}, u_{i+1}\right\}$ and $N\left[u_{j}\right]=\left\{u_{j-1}, u_{j}, u_{j+1}\right\}, 2 \leq i, j \leq n-1$. For $i=1$ or $i=n, N\left[u_{1}\right]=\left\{u_{n}, u_{2}\right\}$ and $N\left[u_{n}\right]=\left\{u_{n-1}, u_{1}\right\}$. Since $\kappa\left(u_{i}\right)=\kappa\left(u_{j}\right)$, from Proposition 2 we obtain that one of the inclusion relations $N\left[u_{i}\right] \subseteq N\left[u_{j}\right]$ or $N\left[u_{i}\right] \supseteq N\left[u_{j}\right]$ must hold in $\bar{G}$. Obviously this is possible if and only if $i=j$, for $n \geq 5$; this is a contradiction to the assumption that $i \neq j$. Thus, no two vertices in a hole take the same color in a colinear coloring. Therefore, $\lambda(\bar{G})=n$. It suffices to show that $\chi(G)<n$. It is easy to see that for the antihole $\bar{C}_{n}$, $\operatorname{deg}(u)=n-3$, for every vertex $u \in V(G)$. Brook's theorem [3] states that for an arbitrary graph $G$ and for all $u \in V(G), \chi(G) \leq \max \{d(u)+1\}=(n-3)+1=$ $n-2$. Therefore, $\chi(G) \leq n-2<n=\lambda(\bar{G})$. Thus the antihole $\bar{C}_{n}$ does not have the $\chi$-colinear property.

We have showed that the graphs $2 K_{2}$ and antihole do not have the $\chi$ colinear property. It follows that any graph that has the $\chi$-colinear property is $\left(2 K_{2}\right.$, antihole)-free and, thus, any graph that has the $\chi$-colinear property is a co-chordal graph.

Although any graph that has the $\chi$-colinear property is co-chordal, the reverse is not always true. For example, the graph $G$ in Figure 2 is a co-chordal graph but it does not have the $\chi$-colinear property. Indeed, $\chi(G)=4$ and $\lambda(\bar{G})=5$. It is easy to see that this graph is also a split graph. Moreover, not any graph that has the $\chi$-colinear property is a split graph, since the graph $C_{4}$ has the $\chi$-colinear property but it is not a split graph. However, there exist split graph which have the $\chi$-colinear property; an example is the graph $C_{3}$. Recall that a graph $G$ is a split graph if there is a partition of the vertex set $V(G)=K+I$, where $K$ induces a clique in $G$ and $I$ induces an independent set; split graphs are characterized as $\left(2 K_{2}, C_{4}, C_{5}\right)$-free graphs.

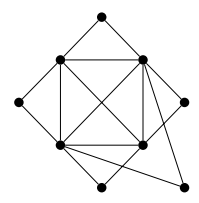

Fig. 2. A graph $G$ which is a split graph but it does not have the $\chi$-colinear property, since $\chi(G)=4$ and $\lambda(\bar{G})=5$ 

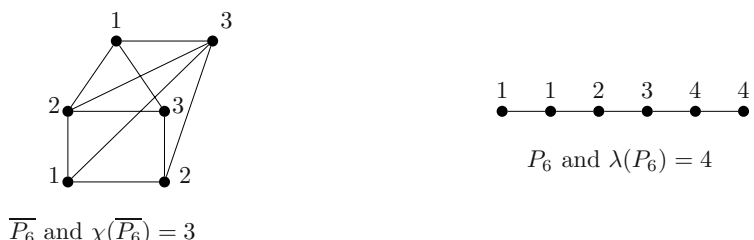

$P_{6}$ and $\lambda\left(P_{6}\right)=4$

$\overline{P_{6}}$ and $\chi\left(\overline{P_{6}}\right)=3$

Fig. 3. Illustrating the graph $\bar{P}_{6}$ which does not have the $\chi$-colinear property, since $\chi\left(\bar{P}_{6}\right) \neq \lambda\left(P_{6}\right)$

We have proved that graphs that satisfy the $\chi$-colinear property do not contain a $2 K_{2}$ or an antihole. Note that, since $\overline{C_{5}}=C_{5}$ and also the chordless cycle $C_{n}$ is $2 K_{2}$-free for $n \geq 6$, it is easy to see that graphs that have the $\chi$-colinear property are hole-free. In addition, the graph $\bar{P}_{6}$ does not have the $\chi$-colinear property (see Figure 3). Thus, we obtain the following result.

Proposition 8. If a graph $G$ satisfies the $\chi$-colinear property, then $G$ is a $\left(2 K_{2}\right.$, antihole, $\left.\bar{P}_{6}\right)$-free graph.

Since graphs having the $\chi$-colinear property are perfect, it follows that any graph $G$ having the $\chi$-colinear property satisfies $\chi\left(G_{A}\right)=\omega\left(G_{A}\right)=\alpha\left(\overline{G_{A}}\right)$, $\forall A \subseteq V(G)$. Therefore, the following result holds.

Proposition 9. A graph $G$ has the $\alpha$-colinear property if and only if the graph $\bar{G}$ has the $\chi$-colinear property.

From Corollary 1] and Proposition 9 we obtain the following characterization.

Proposition 10. The graphs that are characterized by the $\alpha$-colinear property are those graphs $G$ for which the colinear chromatic number achieves its theoretical lower bound in every induced subgraph of $G$.

From Corollary 2 and Proposition 9 we can obtain the following result.

Proposition 11. Any quasi-threshold graph has the $\alpha$-colinear property.

From Propositions 8 and 9 we obtain that graphs that are characterized by the $\alpha$-colinear property are $\left(C_{4}\right.$, hole, $\left.P_{6}\right)$-free. Therefore, the following result holds.

Proposition 12. Any graph that has the $\alpha$-colinear property is a chordal graph.

Although any graph that has the $\alpha$-colinear property is chordal, the reverse is not always true, i.e. not any chordal graph graph has the $\alpha$-colinear property. For example, the complement $\bar{G}$ of the graph illustrated in Figure 2 is a chordal graph but it does not have the $\alpha$-colinear property. Indeed, $\alpha(\bar{G})=4$ and $\lambda(\bar{G})=5$. It is easy to see that this graph is also a split graph. Moreover, not any graph having the $\alpha$-colinear property is a split graph, since the graph $2 K_{2}$ has the $\alpha$-colinear property but it is not a split graph. However, there exist split graphs that have the $\alpha$-colinear property; an example is the graph $C_{3}$. 


\section{Concluding Remarks}

In this paper we introduced the colinear coloring on graphs, proposed a colinear coloring algorithm that can be applied to any graph $G$, and defined two graph properties, namely the $\chi$-colinear and $\alpha$-colinear properties. An interesting question would be to study the graphs that are characterized completely by the $\chi$-colinear or the $\alpha$-colinear property. In addition, it would be interesting to study the relation between the colinear chromatic number and other coloring numbers such as the harmonious number and the achromatic number on classes of graphs.

\section{References}

1. Boesch, F.T., Gimpel, J.F.: Covering the points of a digraph with point-disjoint paths and its application to code optimization. J. of the ACM 24, 192-198 (1977)

2. Brandstädt, A., Le, V.B., Spinrad, J.P.: Graph Classes: A Survey. SIAM, Philadelphia (1999)

3. Brooks, R.L.: On colouring the nodes of a network. Proc. Cambridge Phil. Soc. 37, 194-197 (1941)

4. Chvátal, V., Hammer, P.L.: Aggregation of inequalities for integer programming. Ann. Discrete Math. I, 145-162 (1977)

5. Civan, Y., Yalçin, E.: Linear colorings of simplicial complexes and collapsing. J. Comb. Theory A 114, 1315-1331 (2007)

6. Csorba, P., Lange, C., Schurr, I., Wassmer, A.: Box complexes, neighborhood complexes, and the chromatic number. J. Comb. Theory A 108, 159-168 (2004)

7. Golumbic, M.C.: Algorithmic Graph Theory and Perfect Graphs. Academic Press, New York (1980); annals of Discrete Mathematics, 2nd edn., vol. 57. Elsevier (2004)

8. Hopcroft, J., Karp, R.M.: A $n^{5 / 2}$ algorithm for maximum matchings in bipartite graphs. SIAM J. Computing 2, 225-231 (1973)

9. Kneser, M.: Aufgabe 300. Jahresbericht der Deutschen MathematikerVereinigung 58, 2 (1955)

10. Lovász, L.: Kneser's conjecture, chromatic numbers and homotopy. J. Comb. Theory A 25, 319-324 (1978)

11. Matoušek, J., Ziegler, G.M.: Topological lower bounds for the chromatic number: a hierarchy. Jahresbericht der Deutschen Mathematiker-Vereinigung 106, 71-90 (2004)

12. Nikolopoulos, S.D.: Recognizing cographs and threshold graphs through a classification of their edges. Inform. Proc. Lett. 74, 129-139 (2000)

13. Ziegler, G.M.: Generalised Kneser coloring theorems with combinatorial proofs. Inventiones mathematicae 147, 671-691 (2002) 